ISSN: 0212-5374

DOI: http://dx.doi.org/10.14201/et20143215372

\title{
APRENDIZAJE COLABORATIVO MEDIANTE EL USO DE EDUBLOG EN LA ENSEÑANZA UNIVERSITARIA. VALORACIÓN DE UNA EXPERIENCIA
}

\author{
Collaborative learning and the use of edublogs \\ at university. Assessing an experience
}

\section{Apprentissage collaboratif à travers l'utilisation de l'edublog dans l'enseignement universitaire. Evaluation d'une expérience}

\author{
María D. DAPÍA CONDE* y Ricardo ESCUDERO CID** \\ *Universidad de Vigo. Correo-e: ddapia@uvigo.es \\ ** Universidad Autónoma de Madrid. Correo-e: ricardo.escudero@uam.es
}

Recibido: 15.07.2013; Aceptado: 11.02.2014; Publicado: 31.10.2014

BIBLID [0212-5374 (2014) 32, 2; 53-72]

Ref. Bibl. MARÍA D. DAPÍA CONDE y RICARDO ESCUDERO CID. Aprendizaje colaborativo mediante el uso de edublog en la enseñanza universitaria. Valoración de una experiencia. Enseñanza \& Teaching, 32, 2-2014, 53-72.

RESUMEN: Una de las herramientas colaborativas que ofrece la web 2.0 son los blogs. Por su configuración se convierten en un espacio privilegiado para la comunicación y la construcción del conocimiento grupal. Su introducción en la enseñanza universitaria presencial empieza a hacerse un hueco frente al modelo docente más clásico expositivo y unidireccional. Las investigaciones sobre esta temática se incrementan en el contexto del EEes, que pretende un aprendizaje autónomo, centrado en el alumno, colaborativo y reflexivo. 
El presente trabajo analiza el potencial que tiene la utilización de los edublogs en el contexto de la educación superior, a partir de la evaluación que los/as estudiantes y la docente hacen de un blog grupal en el contexto de la materia Educación para la salud y su didáctica (2.ำ curso del Grado de Educación Infantil). Para la evaluación del mismo se administró un cuestionario adaptado de Durán (2011) a los estudiantes de la materia que asistían regularmente y a la profesora una entrevista acerca de los beneficios y dificultades derivadas del uso del blog. Se presenta un análisis descriptivo de los datos ilustrados con gráficos.

Los resultados revelan un alto grado de satisfacción del alumnado en las escalas consultadas: "dinamización del proceso de enseñanza-aprendizaje", "relaciones personales", "motivación" y "adquisición de contenidos". Asimismo, muestran una alta implicación en la materia, representados en los elevados porcentajes de estudiantes que hacen visitas, leen las entradas o hacen comentarios en el blog. Para la profesora también ha sido una experiencia altamente positiva y enriquecedora; el mayor inconveniente manifestado deriva de la alta dedicación y esfuerzo que exige el mantener la actividad actualizada en la bitácora.

Palabras clave: aprendizaje colaborativo, blogs, docencia, entorno virtual, comunidades virtuales, evaluación, innovación, universidad.

SUMMARY: Blogs are one of the collaborative tools offered by 2.0 web. Their layout makes them a privileged space for communication and group knowledge construction. They are finding a niche in on-site university education against the more classical unidirectional, lecturing teaching model. Research on this subject is increased in the context of the EHEA, which aims at autonomous, student-centered, collaborative and reflective learning.

This current work analyzes the potential of edublogs in the context of higher education, from the evaluation that both students and teacher make of a group blog in the context of the subject of Health Education and its Teaching (2nd year of the Early Childhood Education Degree). For this assessment, an adapted questionnaire from Duran's (2011) was used attending regularly and the teacher an interview about the pros and cons of using a blog. A descriptive analysis of the data illustrated with graphs is presented.

The results reveals a high degree of student satisfaction in the scales checked: "dynamization of the teaching-learning process", "personal relationships", "motivation" and "content acquisition". The results also show high students' involvement in the subject, shown in the high percentage of students who visit, read entries or make comments on the blog. For the teacher it has also been a very positive and rewarding experience; the biggest drawback being the big dedication and effort required to keep the blog updated.

Key words: collaborative learning, blogs, teaching, virtual environment, virtual communities, evaluation, innovation, university. 
RÉSUMÉ: Un des instruments collaboratifs offert par le web 2.0 est le blog. Par sa configuration le blog devient un espace privilégié pour la communication et la construction de la connaissance en groupe. Son introduction dans l'enseignement universitaire "présentiel" commence à se faire une place face au modèle d'enseignement plus classique, expositif et à sens unique. Les recherches à ce sujet augmentent dans le contexte de l'EEEs qui recherche un apprentissage autonome, centré sur l'apprenant, collaboratif et réfléchi.

Dans cette étude nous analysons le potentiel que représente l'utilisation des edublogs dans le contexte de l'enseignement supérieur, à partir de l'évaluation que les étudiants et le professeur font d'un blog en groupe, dans le contexte de la matière Éducation pour la santé et sa didactique (2ème année du "Grado de Educación Infantil»). Pour cette évaluation, on a remis aux étudiants qui assistaient régulièrement aux cours de cette matière un questionnaire adapté de Durán (2011) et au professeur une interview au sujet des bénéfices et des difficultés qui surgissent lors de l'utilisation du blog. Nous présentons une analyse descriptive des données et les illustrons par des graphiques.

Les résultats révèlent le niveau élevé de satisfaction des étudiants dans les catégories consultées: "dynamisation du processus d'enseignement-apprentissage", "relations personnelles", "motivation" et "acquisition de contenus". Ils montrent en outre une forte implication dans la matière, démontrée par les pourcentages élevés d'étudiants qui visitent, lisent les entrées ou font des commentaires sur le blog. Cette expérience a été aussi très positive et enrichissante pour le professeur; le plus grand inconvénient qui a été signalé est que l'actualisation du blog exige beaucoup de temps et d'efforts.

Mots clés: apprentissage collaboratif, blogs, enseignement, environnement virtuel, communautés virtuelles, évaluation, innovation, université.

\section{INTRODUCCIÓN}

La implantación del Espacio Europeo de Educación Superior (EEEs) lleva consigo la modificación de las metodologías y las prácticas de los procesos de enseñanza-aprendizaje más clásicos en el contexto universitario. Frecuentemente, el docente de este nivel fundamenta su rol en la transmisión de conocimientos, basando su metodología de trabajo en la exposición y asumiendo el estudiante un papel pasivo y receptor. La esencia de la Convergencia Europea modifica los roles tradicionales del discente y del docente al centrar el proceso de aprendizaje en el estudiante, reconociendo tanto su trabajo presencial como el no presencial. Este nuevo modelo abandona el enfoque magistral, enciclopédico, transmisivo y da paso a opciones alternativas basadas en un aprendizaje colaborativo, reflexivo, con mayor autonomía, más dinámico, donde el rol del profesor pasa a ser de tutor, orientador, mentor, facilitador del aprendizaje (Aguadez y Pérez, 2007; Blázquez y Alonso, 2009; Merino, López y Ballesteros, 2008; Volman, 2005) y el estudiante asume la responsabilidad de gestionar de manera autónoma su propio aprendizaje (González, García y Gonzalo, 2011). En este proceso un aspecto clave es el aprendizaje colaborativo, que permita un trabajo conjunto (Coutinho, 2007). 
Paralelo al proceso de Convergencia Europea nos acompaña desde hace unas décadas el desarrollo de las nuevas tecnologías de la información y la comunicación, y su aplicación al campo educativo. Ventajas e inconvenientes de esta aplicación han estado presentes en los discursos educativos actuales. Sin entrar en este debate, podemos afirmar que han supuesto el surgimiento de nuevas posibilidades para la práctica docente, aplicadas tanto a la enseñanza presencial como a la no presencial que han influido en la praxis educativa. En algunos casos, han modificado profundamente los modelos de enseñanza-aprendizaje, mientras que, en otras situaciones, las escasas innovaciones se han centrado, de forma única, en la introducción de "nuevas herramientas" sin plantear modificaciones de las prácticas.

Son ya muchos los autores que resaltan la necesidad de incorporar a la enseñanza universitaria las nuevas tecnologías, especialmente algunas herramientas nacidas bajo el amparo de Internet como es el caso de los blogs (Durán, 2011; González, García y Gonzalo, 2011; López, 2009; Williams y Jacobs, 2004). Asimismo, algunos autores (Aguaded y López, 2009; Murga et al., 2008) también ven el blog como una herramienta que facilita la adaptación de la enseñanza universitaria al marco actual de Convergencia Europea, que estimula el desempeño de un rol activo en el alumnado y favorece la evaluación de la propia práctica. La educación actual debe tender a un nuevo modo de construir el conocimiento, romper las barreras espacio-temporales, favorecer el autoaprendizaje y la utilización de metodologías participativas y de colaboración, siendo los edublogs una estrategia que puede responder a estos requerimientos. El valor educativo de un proceso de participación, como el edublog, busca un doble objetivo, por un lado que el alumnado se sienta participe y por otro romper con la unidireccionalidad y la jerarquización del aprendizaje.

\subsection{El blog como herramienta en educación}

Un weblog, blog o bitácora es un sitio web gestionado por uno o más autores reunidos en torno a un tema de interés común (Baltazar, 2005), de fácil uso, compuesto por entradas o posts que se organizan siguiendo una cronología inversa (Huffaker, 2005) y que permite comentarios de los visitantes, por lo que se convierte en una herramienta interactiva.

Existen herramientas, muchas de ellas gratuitas, que permiten administrar un blog. Éstas asignan al usuario una dirección web y le proveen de una interfaz, a través de la cual se puede añadir y editar contenidos. Los edublogs (educación+blogs) son blogs de contenido específicamente educativo, que se utilizan como soporte de los procesos de enseñanza-aprendizaje y que pueden adaptarse a cualquier disciplina y nivel educativo y pueden ser empleados tanto en la enseñanza a distancia como complementarios a la presencial. Actualmente se plantean como una herramienta importante que facilita la aplicación de los 4 pilares de la educación, propuestos por Delors (1996): aprender a ser, a conocer, a hacer y convivir juntos. 
La bibliografía sobre esta temática recoge distintas taxonomías de clasificación, que atienden a criterios diferentes. González y García (2009) plantean una tipología de los blogs educativos, en 4 grupos fundamentales: blogs personales de docentes o expertos en educación, blogs institucionales, blogs de aula y blogs temáticos. Completan esta clasificación con las "comunidades de blogs" que plantean diferenciada por la existencia de interrelaciones con los grupos anteriores. García Manzano (2006) considera como criterio tipológico las diferentes situaciones de enseñanza-aprendizaje y, según éstas, propone los siguientes tipos de edublogs: sistemas de gestión de recursos didácticos, multiblogs de profesores, multiblogs de alumnos, diarios de clase o tutoría y cuadernos de trabajo. A estas cinco clases, Lara (2005) añade una nueva tipología, el blog grupal.

Estas bitácoras virtuales poseen ciertas ventajas para la enseñanza: sencillez de uso (Karpova et al., 2009); manejo de los contenidos de forma eficiente (por temas, por autor, por fecha, etc.); su configuración técnica, basada en el sistema de entradas y comentarios, favorece la interacción y ayuda a crear relaciones; permiten el enlace con otros blogs o mediante hipervínculos; posibilitan la inserción de elementos multimedia (García Manzano, 2006); en la mayoría de las ocasiones utilizan instrumentos gratuitos y de libre acceso; permiten la construcción compartida del conocimiento sobre una cuestión determinada, se aprende "con", "de" y "para" los demás (Salinas y Viticcioli, 2008); facultan la evaluación continua del alumnado y la coevaluación, es decir, la evaluación del trabajo de los estudiantes a través de sus propios compañeros; y amplían los límites espacio-temporales del aula (Salinas y Viticcioli, 2008) pasando a ser el blog un espacio virtual para el autoaprendizaje. Con respecto al aprendizaje autónomo permiten al alumnado "aprender haciendo", además, los links y las etiquetas facilitan la posibilidad de "aprender a aprender", es decir, el estudiante se presenta como un participante activo en su proceso de aprendizaje, lo que implica un mejor desarrollo de sus conocimientos y destrezas. A todo esto se puede añadir una ventaja, poco explotada, como es la flexibilidad para atender la atención a la diversidad de las diferentes situaciones personales del alumnado implicado.

En nuestra experiencia queríamos que el alumnado utilizara los edublogs como fuente de información, medio para la construcción del conocimiento, como canal de comunicación y colaboración entre iguales, como estrategia de reflexión y expresión de opinión, pero sobre todo como una herramienta web bidireccional que permita la retroalimentación (Adell y Castañeda, 2010).

\subsection{Los edublogs, herramientas colaborativas}

Es en los años noventa cuando comienza a plantearse que la tecnología, y sobre todo sus usos, deben incorporar metodologías de orden colaborativo versus cooperativo (Hernández, González y Muchoz, 2014). Es objeto de análisis para diferentes investigadores analizar cuáles son los rasgos distintivos y las posibles diferencias entre colaboración y cooperación. No es nuestra intención en 
este artículo entrar en esta diferenciación prefiriendo hacer hincapié en aquello que tienen en común.

Las herramientas del denominado software social soportado en la plataforma web 2.0 son una excelente opción para el trabajo colaborativo. La web 2.0 también llamada "colaborativa" se considera una web social y participativa, un espacio de trabajo para la actividad grupal, que permite crear, colaborar y compartir conocimientos con los demás; en definitiva un medio de participación, comunicación y trabajo en equipo. Diferentes investigaciones se manifiestan en este sentido y plantean las distintas posibilidades de las web 2.0 (y en concreto de los blogs) como facilitadores y potenciadores del aprendizaje colaborativo (Álvarez, Ayuste, Gros, Guerra y Romañá, 2005; Álvarez y Bassa, 2013; Carrió, 2007; Echazarreta, Prados, Poch y Soler, 2009; Fernández y Valverde, 2014; Hernández, González y Muñoz, 2014; Levis, 2011; Rebollo, García, Buzón y Barragán, 2012; Ruiz y Abella, 2011; Sánchez, 2011). Las razones son varias; permiten una comunicación en varias direcciones, un proceso con experiencias y aprendizaje compartido y, por tanto, una elaboración compartida del conocimiento (Álvarez y Bassa, 2013). Proporcionan oportunidades para el desarrollo de habilidades sociales y comunicativas, actitudes positivas hacia las personas, cohesión grupal y construcción de relaciones sociales (Fernández y Valverde, 2014) y como indica Carrió (2007: 5): «El fortalecimiento de la autoestima al ser aceptadas las ideas que se comparten y que los demás miembros aceptan como válidas, por lo que se consigue un aprendizaje conceptual y social al mismo tiempo".

El desarrollo de proyectos colaborativos puede experimentar un notable impulso con la utilización de las Tic (Echazarreta, Poch y Soler, 2009), beneficiándose en los siguientes aspectos (Calzadilla, 2001: 8):

a) Estimulación de la comunicación interpersonal en el aprendizaje virtual ya que posibilita el intercambio de información de los miembros del grupo involucrado.

b) Permitir que los estudiantes compartan información, trabajen con documentos conjuntos, ficheros, notas, foros de discusión, etc., y facilitar la solución de problemas y toma de decisiones.

c) Seguimiento eficaz del progreso del grupo, a nivel individual y colectivo.

d) Acceso rápido a la información y los contenidos del aprendizaje.

e) La gestión y administración de los alumnos es más sencilla ya que disponemos de todos sus datos y que pueden ser de utilidad en momentos concretos.

f) Creación de ejercicios de evaluación y autoevaluación, con los que el docente podrá conocer el nivel de logro y rediseñar la experiencia de acuerdo a su ritmo y nivel y al estudiante le ofrecerán retroalimentación.

A todo lo anterior podemos añadir otras características señaladas por Carrió (2007): posibilidad de utilizar experiencias anteriores; difusión de las experiencias 
y poder contactar con otros; investigar sobre distintos logros con otros grupos aunque estén en lugares muy distintos; existencia una gran flexibilidad cognitiva, y, por último, el aprendizaje colaborativo asistido por ordenador facilita la tarea para aquellos miembros que prefieren no enfrentarse a las clases presenciales con el grupo y se deciden por el trabajo remoto.

Las implicaciones para la docencia de la introducción de los recursos sociales en la búsqueda de un aprendizaje colaborativo en el ámbito escolar de cualquier nivel (y de forma más notoria en el universitario) son diversas, implicando cambios sustanciales en el modelo de enseñanza-aprendizaje, en el rol del docente y del estudiante. El modelo educativo se centra en el alumno, del que se exige una implicación responsable en el proceso y que, según la definición de aprendizaje colaborativo propuesta por Begoña Gros (2008), el estudiante está en interacción con los demás y no se considera un individuo aislado. El rol del docente también se ve alterado; desaparece el formato tradicional profesor-emisor, y por ende la función básica de transmisión unilateral, para ejercer una función de acompañamiento, guía, fomentando la construcción e intercambio de conocimientos (Levis, 2011; Gros, 2007, 2008).

Una parte importante de su intervención se centra en la retroalimentación y el seguimiento de las tareas de aprendizaje, reforzando acciones positivas del alumnado y orientando el proceso de aprendizaje (Rebollo, García, Buzón y Barragán, 2012: 123).

\subsection{Contextualización y descripción de la experiencia}

Este trabajo tiene como objetivo fundamental poner de manifiesto el potencial del uso de los edublogs en la enseñanza superior. Para ello se ha diseñado una experiencia consistente en la creación y el mantenimiento de una comunidad de aprendizaje virtual a través de la utilización del edublog Educación para a saúde en infantil (Educación para la salud en infantil) http://educacionparaasaudeinfantil.blogspot.com). Ésta fue la herramienta fundamental de la asignatura Educación para la Salud y su Didáctica, del segundo curso del Grado de Educación Infantil de la Universidad de Vigo (Facultad de Ciencias de la Educación, Campus de Ourense), en la que se combinó la enseñanza presencial y la virtual.

En la primera clase del curso se formularon cinco preguntas a los estudiantes con el fin de conocer los recursos y los conocimientos previos. El 100\% del alumnado tenía ordenador y el 93,5\% tenía conexión a Internet. En lo que se refiere a los blogs, el 85\% conocía lo que era un blog, sólo el 37\% había participado antes en alguno y el 33\% afirmaba saber diseñarlo y configurarlo.

A tenor de estos resultados, se puede afirmar que los estudiantes son especialmente receptivos ante el uso de las nuevas tecnologías, pero esto no quiere decir que todos estén capacitados digitalmente para el uso y aprovechamiento de todas las herramientas. Por ello, a pesar de la sencillez de uso de los blog y siguiendo 
las recomendaciones de González, García y Gonzalo (2011), al comienzo del curso, realizamos dos sesiones de laboratorio con el fin de que todo el alumnado conociera el funcionamiento y las posibilidades que este sitio web ofrece. En estos primeros encuentros se animó a los estudiantes a que fueran creativos y se estimuló la interactividad, es decir, el establecimiento de conexiones virtuales entre la profesora y el alumnado y de éstos entre sí. Estas sesiones se reforzaron de forma continuada mediante la tutoría cuando se realizaban tareas nuevas.

El modelo seguido fue un edublog gestionado por la profesora, al que se insertaron las doce bitácoras de los doce grupos formados, según preferencias, por cuatro o cinco estudiantes (Tabla 1). Este blog estaría encuadrado en la tipología blog grupal. Cada uno de los blogs de los equipos de estudiantes trabajó uno de los contenidos tópicos de educación para la salud: alimentación, higiene, prevención de accidentes, educación emocional, educación afectivo-sexual y prevención de alcohol y tabaco.

Cada uno de los blogs adopta un modelo didáctico centrado en el estudiante, ya que se fundamenta en la construcción del aprendizaje a partir de la participación activa del alumnado y el intercambio colaborativo con los demás miembros: docente, componentes del equipo y de los otros blogs.

Una vez configurados los distintos blogs se publicaron en ellos las actividades propuestas por la profesora a lo largo del curso, unas de carácter individual y otras realizadas por todo el equipo responsable de cada blog. Dichas actividades hacían referencia a búsqueda de noticias relacionadas con el tema, de vídeos, elaboración de material didáctico... A partir de la activación de los distintos blogs, el número de visitas a los mismos contabilizadas al finalizar el cuatrimestre fue alto -entre 445 y 1230 - (ver Tabla 1$)$.

El «blog de blogs" se constituyó como una plataforma común que permitió: la comunicación bidireccional entre estudiante-profesora y estudianteestudiante, el aprendizaje compartido y la evaluación continua del trabajo del alumnado.

Debido a que el desarrollo de cada bitácora sería tenido en cuenta en la evaluación de la materia, las últimas sesiones de clases presenciales de la materia se destinaron a que todos los estudiantes participasen en la evaluación de cada uno de los blogs; una coevaluación que buscaba reflexionar acerca del trabajo realizado los diferentes grupos, entendida ésta como un proceso de mejora. 
MARÍA D. DAPÍA CONDE Y RICARDO ESCUDERO CID

TABLA 1

Títulos de los blogs y número de visitantes durante el cuatrimestre

\begin{tabular}{|l|c|}
\hline \multicolumn{1}{|c|}{ Título DE LOS BLOGS DEL ALUMNADO } & $\begin{array}{c}\text { N.. DE VISITAS AL } \\
\text { FINALIZAR EL CUATRIMESTRE }\end{array}$ \\
\hline $\begin{array}{l}\text { A alimentación: aprendendo a comer (La alimentación: } \\
\text { aprendo a comer) }\end{array}$ & 445 \\
\hline $\begin{array}{l}\text { A hixiene: limpeza e coidado do noso corpo (La higiene: } \\
\text { limpieza y cuidado de nuestro cuerpo) }\end{array}$ & 545 \\
\hline $\begin{array}{l}\text { Accidentes frecuentes nos nenos e nenas } \\
\text { (Accidentes frecuentes en nuestros niños y niñas) }\end{array}$ & 790 \\
\hline Por unha infancia sen fume (Por una infancia sin humo) & 502 \\
\hline Alcohol na etapa infantil (Alcohol en la etapa infantil) & 998 \\
\hline $\begin{array}{l}\text { Educación afectivo sexual na educación infantil (Educación } \\
\text { afectivo sexual en la educación infantil) }\end{array}$ & 788 \\
\hline Educar as emocións (Educar las emociones) & 821 \\
\hline Security children & 1230 \\
\hline ¡Aliméntate connosco! (Aliméntate con nosotros) & 696 \\
\hline Mergúllate na saúde (Sumérgete en la salud) & 846 \\
\hline Pequeño trasno sen fume (Pequeño trasno sin humo) & 722 \\
\hline ¡Riscos do alcohol! (Riesgos del alcohol) & \\
\hline
\end{tabular}

(Traducción de títulos originales del idioma gallego al castellano).

\section{Metodología}

Esta investigación se planteó como objetivo conocer la valoración que estudiantes y docente hacen del uso del blog en una materia universitaria, en relación al fomento de la participación, la dinamización del proceso de enseñanza-aprendizaje, las relaciones sociales, la motivación y la adquisición de contenidos en su formación como futuros profesores.

Para la evaluación del "blog de blogs" se administró un cuestionario anónimo a los estudiantes de la asignatura, que asistían regularmente a clase, adaptado de Durán (2011). El cuestionario estaba constituido por 46 ítems tipo Likert agrupados en cinco escalas: participación, dinamización del proceso de enseñanza-aprendizaje, relaciones personales, motivación y adquisición de contenidos. Cada uno de los ítems ofrece 4 opciones de respuesta, con la intención de obligar al estudiante a inclinarse en una dirección su valoración. Las cuatro respuestas posibles son: totalmente de acuerdo, medianamente de acuerdo, medianamente en desacuerdo y totalmente en desacuerdo. El cuestionario se completaba con 4 cuestiones de valoración global del blog y de su uso, que los estudiantes tenían que responder 
completando una escala de puntuación entre 0 (valor negativo mínimo) y 10 (valoración positiva máxima).

Los cuestionarios fueron contestados de forma voluntaria y anónima por los estudiantes. En total, los estudiantes que han participado y colaboraron en la investigación fueron 58 .

Para el análisis de datos se acudió principalmente a la utilización de técnicas de estadística descriptiva univariada, fundamentalmente porcentajes y estadísticos de tendencia central (medias) mediante el programa estadístico SPSS 19.0 para Windows.

La valoración de la profesora se realizó a partir de una entrevista abierta con dos cuestiones referidas a los beneficios y dificultades detectadas en el uso del blog, atendiendo particularmente a los cinco tópicos que constituían las escalas del cuestionario de los estudiantes.

\section{RESUltados: VALORACIÓN DEL EDUBLOG}

Las cifras obtenidas en el cuestionario de los estudiantes refieren resultados muy favorables y un alto grado de satisfacción en los cinco tópicos (escalas) consultados.

En la escala 1 (Gráfico 1) se valoró la participación del alumnado teniendo en cuenta las visitas, entradas, lecturas y comentarios en el blog. Los estudiantes indicaron que visitaron muchas o bastantes veces tanto el blog de la profesora, como el de su equipo o el de sus compañeros en un $89,1 \%, 100 \%$ y $82,7 \%$ respectivamente. Así mismo, en la Tabla 1 queda reflejado el elevado número visitas de cada blog al terminar el periodo lectivo, detectándose un alto grado de participación.

Esta participación puede considerarse bastante completa. Casi el 90\% de los encuestados subieron entradas al blog de su equipo y leyeron el de la profesora y el de su equipo muchas o bastantes veces. Sin embargo, el porcentaje de los comentarios se reduce a la mitad o incluso menos con respecto a los que dicen leerlos mucho/bastante. Es curioso que el $30 \%$ de los estudiantes afirmen no comentar nunca las noticias de sus compañeros de equipo. Por otra parte, los blogs menos leídos fueron los del resto de los compañeros, indicando casi el 35\% que los leyeron pocas veces.

En el análisis de la escala 2 (Gráfico 2) podemos destacar la excelente valoración que los estudiantes hacen del edublog como instrumento dinamizador del proceso de enseñanza-aprendizaje; así, supera el 90\% los que indican que estimula el seguimiento de la asignatura, estimula la búsqueda de información nueva sobre el tema, facilita la expresión de ideas y opiniones sobre temas de actualidad relacionados con la asignatura, fomenta la participación de los estudiantes en la asignatura, fomenta el pensamiento crítico y favorece el pensamiento creativo. El ítem "el blog favorece la solución de dudas en cuanto a la asignatura" recibe una valoración menos alta, aunque con tendencia positiva; el 30,4\% expresa estar medianamente en desacuerdo con tal proposición. 


\section{GRÁFICO 1}

Porcentaje de participación de los estudiantes en el blog

(visitas, entradas, lectura y comentarios)

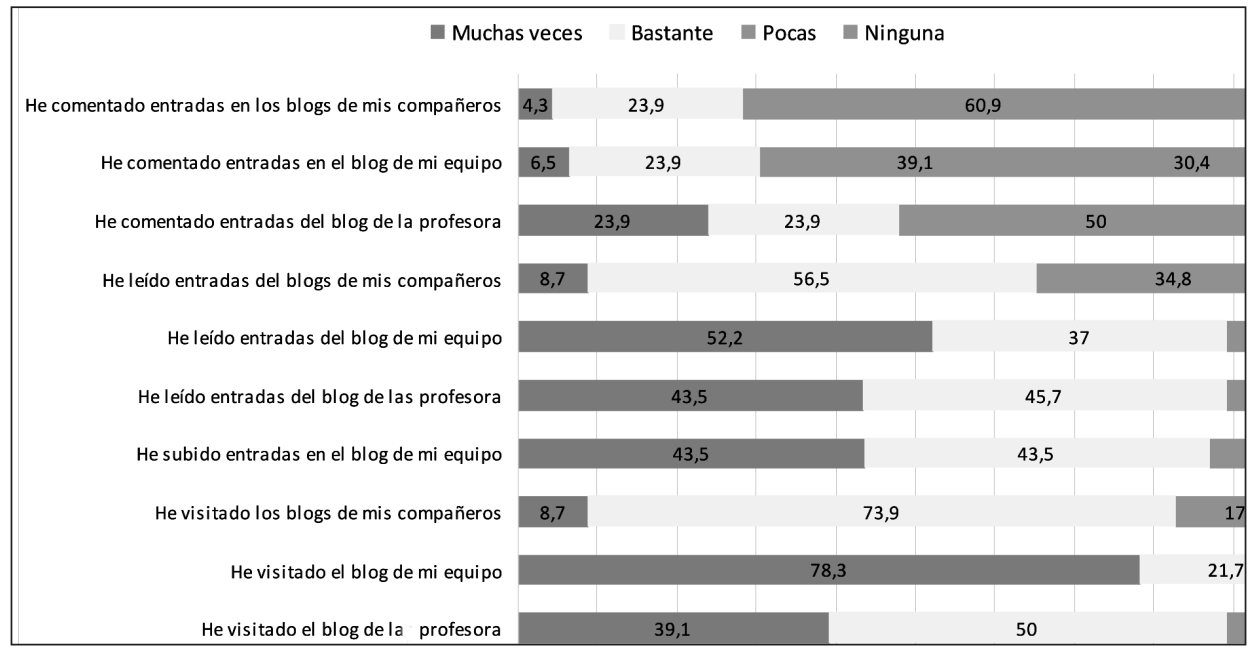

\section{GRÁFICO 2}

Valoración escala "dinamización del proceso de enseñanza-aprendizaje" (en porcentajes)

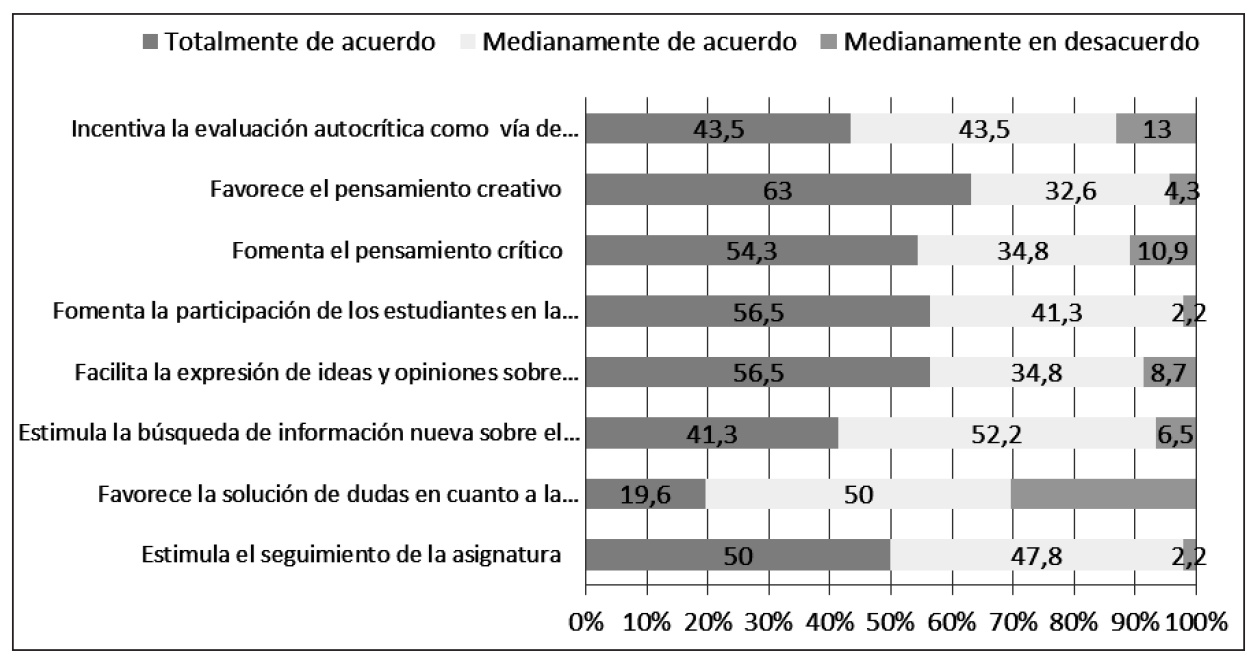


El sistema educativo tradicional ha limitado el desarrollo de las competencias sociales y de pensamiento crítico. El análisis de la escala 3 relativa a las relaciones sociales (Gráfico 3) marca una tendencia contraria, indicando de forma clara que los estudiantes perciben que el edublog favorece las relaciones personales, estableciendo nuevas modalidades de relación docente-alumnado, así como incrementando las posibilidades de contacto entre el grupo de estudiantes. Así, superan el 80\% los estudiantes que manifiestan estar total o medianamente de acuerdo en proposiciones tales como: facilita el contacto con la profesora; potencia la colaboración con la profesora; facilita la comunicación entre los estudiantes; fomenta las relaciones personales con los compañeros; promueve que el estudiante se sienta parte del grupo; estimula nuevas formas de relación. Destaca que el 93,5\% percibe que el blog favorece la interacción y el trabajo colaborativo del alumnado.

GRÁFICO 3

Valoración escala "relaciones personales" (en porcentajes)

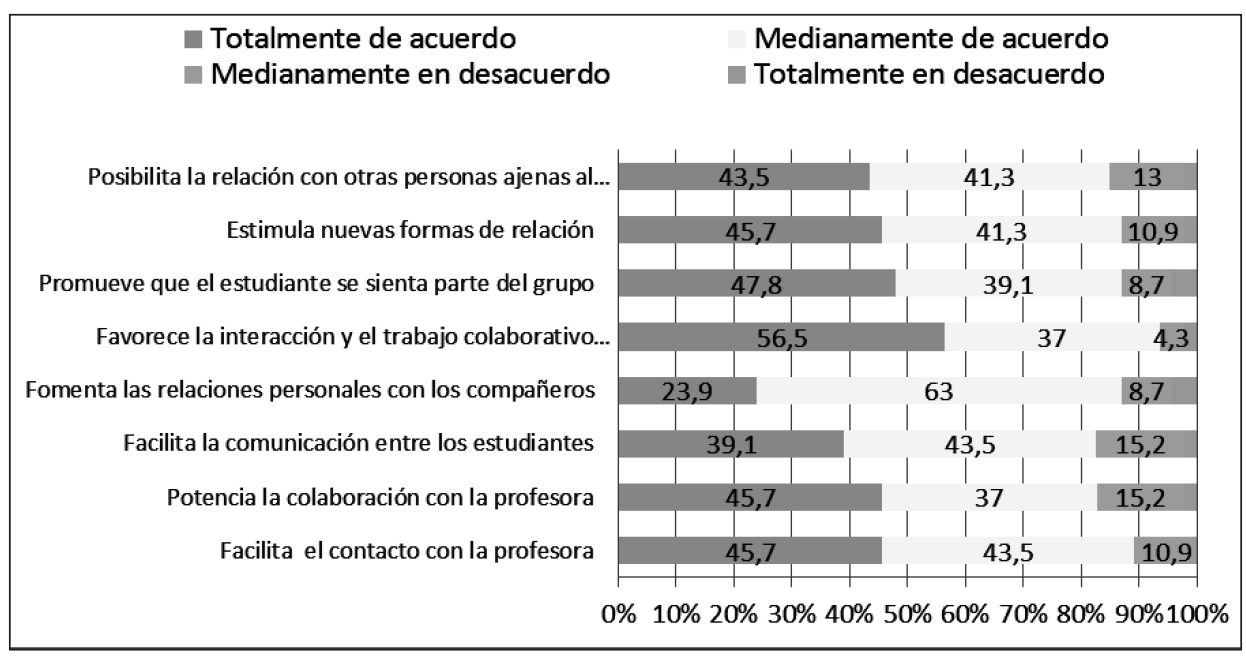

El Gráfico 4 representa los resultados de los estudiantes ante el uso del edublog como herramienta que favorece la motivación (escala 4). Ninguna de las proposiciones incluidas obtiene valoración negativa, presentando, por el contrario, una tendencia claramente positiva en relación con fomentar el interés por llevar hábitos saludables, profundizar en el tema, realizar actividades de la asignatura, usar las nuevas tecnologías...

Con la última escala, se pretende conocer la idoneidad del edublog en la adquisición de contenidos. Otro de los aspectos positivos, como puede apreciarse en el Gráfico 5, del uso de este recurso en la docencia es que facilita la adquisición de contenidos específicos de la materia, tanto teóricos como prácticos; actitudes positivas ante las innovaciones educativas y la práctica como futuros docentes; 
el desarrollo de conocimientos menos específicos (relativos a las Nuevas Tecnologías), y, algo fundamental, en esta sociedad de la información, es que para el 98,8\% favorece la construcción de nuevos conocimientos, nuevos pensamientos.

GRÁFICO 4

Valoración escala "motivación" (en porcentajes)

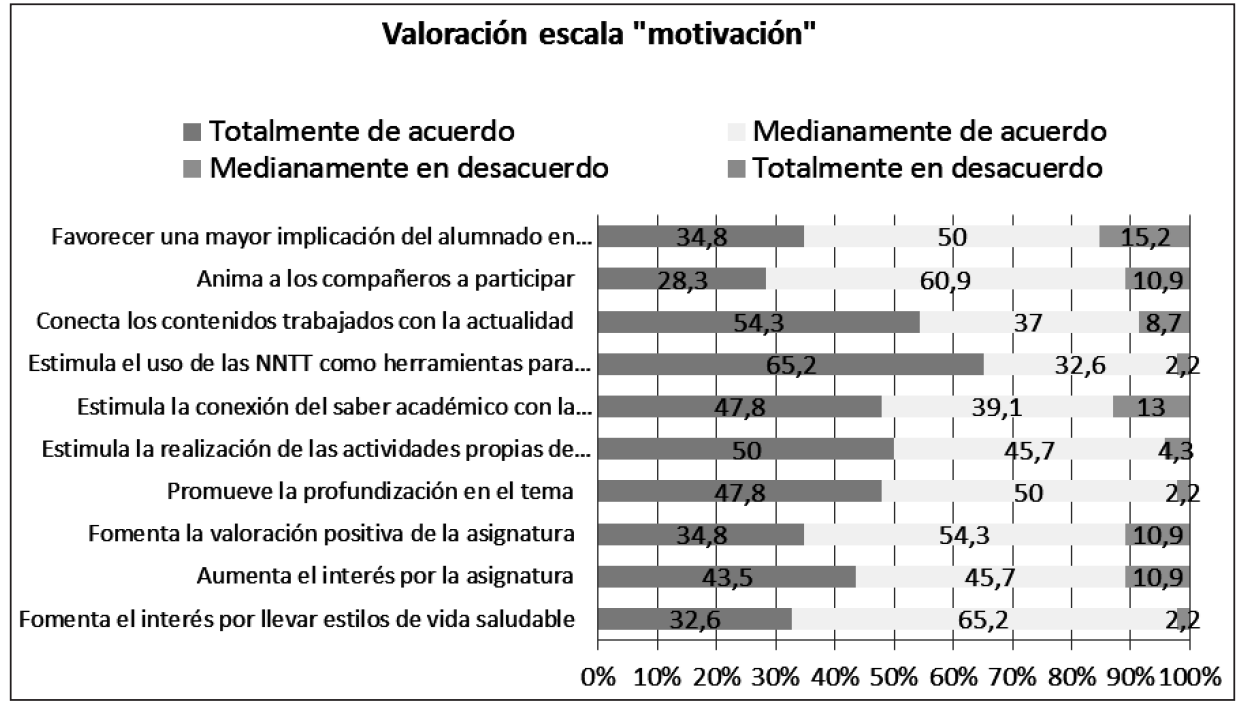

GRÁFICO 5

Valoración escala "adquisición de contenidos" (en porcentajes)

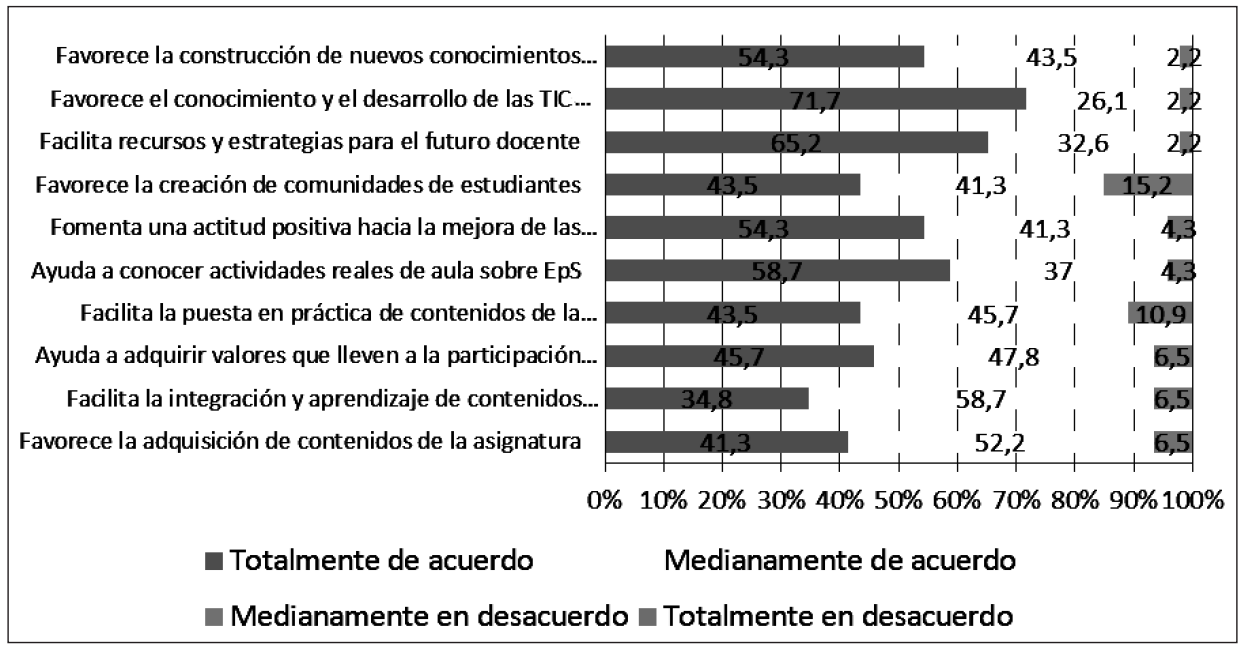


Por último, respecto a las cuestiones globales acerca del blog y de su uso (en una escala de 0 a 10) los estudiantes hacen una notable valoración del blog como herramienta $(\overline{\boldsymbol{x}}=7,8)$; califican de forma destacable $(\overline{\boldsymbol{x}}=8,6)$ el uso que hizo la profesora del blog y valoran su participación con un 7,3. Por último, comprobamos que consideran positivo $(\overline{\boldsymbol{x}}=8,1)$ el interés que para ellos tienen los edublogs en la práctica docente.

La profesora valora positivamente la experiencia realizada, opina que los estudiantes han mejorado competencias como: mayor interés por la Educación para la salud, mejor capacidad de autoaprendizaje y mayor responsabilidad. Reconoce la existencia de aprendizaje significativo y afirma que permitió mejorar las capacidades no solo del alumnado sino también de ella misma.

La Red es un excelente recurso para favorecer las relaciones personales, la comunicación, el intercambio de información. El formato blog permite la interacción didáctica virtual entre el docente y el estudiante, así como entre éste y sus pares. Los posts y los comentarios característicos de los blogs amplían las posibilidades de comunicación y generan nuevas oportunidades de aprendizaje.

En relación a la evaluación, la profesora valora positivamente que el blog le permita visualizar el recorrido de construcción del aprendizaje de los estudiantes, lo que le facilita una retroalimentación del proceso. Además, la presencia pública en la Red de los trabajos de los estudiantes facilita la coevaluación; esta forma innovadora de evaluar fomenta en el alumnado que se sientan partícipes del proceso educativo, haciendo juicios críticos acerca del trabajo de sus compañeros y estimula la responsabilidad, ya que les hace conscientes de cómo impacta su trabajo en el resultado final de las tareas colaborativas.

Valora positivamente el poder ampliar las horas presenciales de la asignatura, pero esto, que es considerado, en principio, como positivo, se convierte a la vez en la mayor dificultad. La exigencia de una elevada constancia en el mantenimiento de la bitácora requiere del esfuerzo y de la responsabilidad de todos: docente y discentes. Para que el edublog funcione, como comunidad de aprendizaje de forma eficiente, es imprescindible la acción docente en un nuevo escenario virtual. La ratio de alumnado y el elevado número de blogs hicieron que el trabajo resultara muy fatigoso, siendo una de las principales dificultades del uso de este nuevo soporte educativo. Por otro lado, la libertad de funcionamiento de las nuevas comunidades virtuales hace que aparezcan nuevas responsabilidades docentes, para intentar evitar que surjan actuaciones no esperadas o desfavorables, como: utilizar la metodología del "Corta y pega"; no citar las fuentes de documentación; insertar comentarios fuera de lugar, etc.

\section{DISCUSIÓN Y CONCLUSIONES}

Los estudiantes universitarios actuales pertenecen a los denominados "nativos digitales" (Prensky, 2001a y b), caracterizados por su habilidad y aptitud para contextos como Facebook, Tuenti, Twitter, descargas de música o vídeo, etc., sin 
embargo, esta experiencia indica que para la mayoría de los estudiantes, cuando se alejan de las redes sociales, la competencia en las nuevas tecnologías dista de ser la deseada. En esta misma línea, Salinas y Viticcioli (2008), en una investigación con universitarios, refieren, en relación a su experiencia previa con blogs, que sólo el $25 \%$ tenía experiencia como participante activo de un blog (16\% como creadores y 10\% como comentaristas), si bien el 73\% declaró conocer qué era un blog.

La experiencia de introducir un blog en una materia universitaria en el marco del EEes, y asumiendo como modelo de trabajo el aprendizaje colaborativo, ha sido positiva tanto para los estudiantes como para docentes, promoviendo un elevado nivel de implicación del alumnado, a tenor de los altos porcentajes de estudiantes que hacen visitas, insertan entradas o hacen comentarios en el blog. Las 5 escalas objeto de valoración ("participación", "dinamización del proceso de enseñanzaaprendizaje", "relaciones interpersonales", "motivación" y "adquisición de contenidos") han recibido una alta aprobación, reforzando resultados de estudios previos realizados sobre el tema, particularmente los que a continuación se mencionan.

En primer lugar, el uso del edublog contribuyó a generar procesos de participación, suponiendo altas tasa de participación de los estudiantes; el empleo del blog como recurso educativo ha producido impacto, ya que como refieren Ferdi y Trammel (2004) les abre y acerca canales de participación y les ofrece nuevas perspectivas dentro y fuera del aula.

En segundo lugar, puede considerarse una práctica adecuada para mantener un papel activo del estudiante (Cabero, López y Ballesteros, 2009), permitiendo la reflexión y el contraste de ideas propias y ajenas (Bohórquez, 2008) y facilitando que los estudiantes sean más autónomos (Bhattacharya y Chauhan, 2010).

En tercer lugar, la experiencia también fue favorable para el desarrollo de competencias sociales (aprender a convivir juntos) en la construcción colaborativa del conocimiento. La adquisición de las mismas puede plantearse como uno de los logros más apreciados en el mercado laboral actual. Los blogs son una herramienta de colaboración y comunicación (Achterman, 2006; Cuhadar y Kuzu, 2010; López, 2009; Marquès, 2007; Ray, 2006). Sin embargo, los estudiantes siguen prefiriendo una comunicación directa frente a la derivada del weblog (Andergassen et al., 2009).

En cuarto lugar, el edublog se ha convertido en un instrumento de fácil uso que incrementó la motivación (Chain, Martínez y Sánchez, 2008). Los estudiantes perciben beneficios en el uso del blog ya que sus intereses y motivación se incrementan debido a la interacción y feedback con sus compañeros y profesores (Aljumah, 2012).

En quinto lugar, tampoco se pueden obviar los beneficios de orden cognitivo; el blog facilitó la adquisición de contenidos, contribuyendo a mejorar la comprensión y el dictado de la materia, como en otros estudios (Chain, Martínez y Sánchez, 2008; Salinas y Viticcioli, 2008), sirviendo como apoyo a la docencia (Cabero, López y Ballesteros, 2009). Además, no podemos olvidar como indican Camilli, López y Barceló (2012) que el aprendizaje cooperativo se convierte en un indicador estimable y predictor de buenos resultados. 
A tenor de los resultados, la utilización de los edublogs puede significar un gran paso para superar algunos obstáculos que arrastra la enseñanza en general y la universitaria en particular: falta de motivación del alumnado, enseñanza pasiva, enseñanza centrada en el aprendizaje de conocimientos teóricos descontextualizados. La eficacia real de los edublogs como herramienta de aprendizaje dependerá de la motivación y la formación del docente, de la importancia y el valor real que se le dé desde el sistema educativo a la innovación y, por supuesto, a los esfuerzos dedicados a mejorar los recursos necesarios. Consideramos el edublog una herramienta adecuada para superar paradigmas centrados en la lección magistral y promover la participación, la interacción y la colaboración como bases del aprendizaje para la innovación y la mejora de la calidad de los procesos de enseñanzaaprendizaje. Asimismo, puede considerarse un instrumento potencialmente adecuado que permite promover el "aprendizaje autónomo" (González y García, 2009; Murga et al., 2008), principio básico de las exigencias en la actual implantación del Espacio Europeo de Educación Superior (EEES) y que produce un alto grado de satisfacción en los estudiantes.

Sin embargo, no podemos finalizar sin aludir a algunas dificultades acerca de la introducción del aprendizaje colaborativo en la enseñanza señaladas en investigaciones anteriores (Álvarez, Ayuste, Gros, Guerra y Romañá, 2005; Gros, 2007, 2008; Levis, 2011; Sánchez, 2011). A pesar de las virtualidades que ofrece la web 2.0 como entorno colaborativo de aprendizaje y comunicación, el sistema educativo favorece la competencia y el logro individual, por lo que los estudiantes manifiestan reticencias a participar en procesos colaborativos. Además cambiar el modelo transmisor dominante por el de "constructor" del conocimiento tampoco es una preferencia ni de estudiantes ni de docentes en un contexto desfavorable per se. Los desafíos para el docente incluyen el conocimiento del entorno virtual y la comprensión de los objetivos del aprendizaje colaborativo (Scagnoli, 2005).

Tampoco podemos olvidar que la calidad educativa no reside en la innovación tecnológica sino en el propio proceso de enseñanza-aprendizaje desencadenado (Levis, 2011). Las tecnologías multimedia e Internet se han incorporado paulatinamente a las aulas. A menudo, su uso, tanto por parte del alumnado como del profesorado, se muestra como un elemento reforzador de las prácticas educativas tradicionales. Si realmente queremos conseguir una enseñanza innovadora mediante las TIC debemos aprovechar la potencialidad de estas tecnologías para impulsar nuevas formas de aprender y enseñar. El profesorado puede utilizar las nuevas tecnologías para propiciar el autoaprendizaje, el aprendizaje colaborativo, el diálogo y la reflexión... y no hacer un uso de estos recursos solamente como fuente de información; no se trata de hacer lo mismo sobre nuevos soportes, sino desarrollar nuevas estrategias didácticas.

Somos conscientes de las limitaciones de esta investigación: número de sujetos limitado, una sola docente, exclusivamente estudiantes de la carrera de formación del profesorado... y por ello se hace necesario ampliar las investigaciones en esta línea, que tenga en cuenta aspectos tales como el estilo docente, uso de blog en 
el modelo pedagógico, características de los estudiantes, nivel educativo, antecedentes de utilización... También parece necesario profundizar en algunos de los inconvenientes del uso del edublog que pueden derivarse de este estudio: la «inversión" de trabajo y tiempo que dedica el profesor en la revisión de los posts y de los enlaces; el uso de la tecnología como herramienta y no como meta en sí misma en los procesos de enseñanza-aprendizaje; las modificaciones de las prácticas educativas con el uso de los blogs; las potencialidades versus usos de los edublogs...

\section{REFERENCIAS BIBLIOGRÁFICAS}

Achterman, D. (2006). Making connections with blogs and wikis. California School Library Association Journal, 30, 1, 29-31.

Adell, J. y Castañeda, L. J. (2010). Los Entornos Personales de Aprendizaje (PLEs): una nueva manera de entender el aprendizaje. En R. I. Roig Vila y M. Fiorucci (coords.). Claves para la investigación en innovación y calidad educativas, la integración de las tecnologías de la información y la comunicación y la interculturalidad en las aulas (pp. 19-33). Alcoy: Marfil.

Aguaded, J. I. y López Meneses, E. (2009). La blogosfera educativa: nuevos espacios universitarios de innovación y formación del profesorado en el contexto europeo. REIFOP, $12,3,165-172$. http: //www.aufop.com/aufop/revistas/arta/digital/138/1263.

Aguaded, J. I. y Pérez, M. (2007). La educación en medios de comunicación como contexto educativo en un mundo globalizador. En J. Cabero (Coord.). Nuevas tecnologías aplicadas en la educación (pp. 63-75). Madrid: McGraw-Hill.

Aljumah, F. H. (2012). Saudi learner perceptions and attitudes towards the use of blogs in teaching English writing course for EFL Majors at Qassim University. English Language Teaching, 5, 1, 100-116.

Álvarez, I.; Ayuste, A.; Gros, B.; Guerra, V. y Romañá, T. (2005). Construir conocimiento con soporte tecnológico para un aprendizaje colaborativo. Revista Iberoamericana de Educación, 36, 1. http: //www.rieoei.org/tec_edu37.htm.

Álvarez, G. y Bassa, L. (2013). TIC y aprendizaje colaborativo: el caso de un blog de aula para mejorar las habilidades de escritura de estudiantes preuniversitarios. Revista de Universidad y Sociedad del Conocimiento, 2, 10, 5-19. http://dx.doi.org/10.7238/rusc.v10i2.1740.

Andergassen, M.; Behringer, R.; Finlay, J.; Gorra, A. y Moore, D. (2009). Weblogs in Higher Education: Why Do Studenst (Not) Blog? Electronic Journal of e-Learning, 7, 3, 203-214.

Baltazar, N. (2005). Weblogues: um novo instrumento para a promoção da comunicação entre televisão e telespectadores. Comunicar, 25, 2.

Bhattacharya, A. y Chauhan, K. (2010). Augmenting Learner Autonomy through Blogging. ELT Journal, 64, 4, 376-384. http: /dx.doi.org/10.1093/elt/ccq002.

Blázquez, F. y Alonso, L. (2009). Funciones del profesor de e-learning. Pixel Bit. Revista de Medios y Educación, 34, 205-215.

Bohórquez, E. (2008). El blog como recurso educativo. EDUTEC, Revista Electrónica de Tecnología Educativa, 26. 
http:V/edutec.rediris.es/Revelec2/revelec26/edutec26_el_blog_como_recurso_educativo.html

Cabero, J.; López, E. y Ballesteros, C. (2009). Experiencias universitarias innovadoras con blogs para la mejora de la praxis educativa en el contexto europeo. Revista de Universidad y Sociedad del Conocimiento, 6, 2. http://rusc.uoc.edu/ojs/index.php/rusc/article/view/v6n2_cabero_etal/v6n2_cabero

Calzadilla, M. E. (2001). Aprendizaje colaborativo y tecnologías de la información y comunicación. Revista Iberoamericana de Educación, 1, 10. http: //www.rieoei.org/delos lectores/322Calzadilla.pdf

Camilli, C.; López, E. y Barceló, M.ํㅜ L. (2012). Eficacia del aprendizaje cooperativo en comparación con situaciones competitivas o individuales. Su aplicación en la tecnología: Una revisión sistemática. Enseñanza \& Teaching, 30, 2, 81-102.

Carrió, M. (2007). Ventajas del uso de las tecnologías en el aprendizaje colaborativo. Revista Iberoamericana de Educación, 41, 4. http://www.rieoei.org/deloslectores/1640Carrio.pdt

Chain, C.; Martínez, L. y Sánchez, J. J. (2008). Motivar desde la innovación en la enseñanza universitaria: El blog Qalidad. RED. Revista de Educación a Distancia, 21.

Coutinho, C. (2007). Cooperative Learning in Higher Education using Weblogs: a study with undergraduate Education in Portugal. World Multiconference on Systemics, Cybernetic and Informatics, 11, 1, 60-64.

Cuhadar, C. y Kuzu, A. (2010). Improving Interaction through Blogs in a Constructive Learning Environment. Turkish Online Journal of Distance Education, 11, 1, 134-161.

Delors, J. (1996). La educación encierra un tesoro. Madrid: Santillana. Ediciones UNESCO.

Durán, J. F. (2011). La contribución del edublog como estrategia didáctica. Electronic Journal of Research in Educational Psychology, 9, 1, 331-356. http: //wWw.investigacionpsicopedagogica.org/revista/articulos/23/espannol/ Art_23_494.pdf

Echazarreta, C.; Prados, F.; Poch, J. y Soler, J. (2009). La competencia «El trabajo colaborativon: una oportunidad para incorporar las TIC en la didáctica universitaria. Descripción de la experiencia con la plataforma ACME (UdG). Uocpapers, 8. 1-11. http: //www.uoc.edu/uocpapers/8/dt/esp/echazarreta_prados_poch_soler.pdf

Ferdi, R. y Trammel, K. (2004). Content Delivery in the Blogosphere. T. H. E. Journal Online.

http://www.thejournal.com/magazine/vault/articleprintversion.cmf?aid = 4677

Fernández, M. R. y Valverde, J. (2014). Comunidades de práctica: un modelo de intervención desde el aprendizaje colaborativo en entornos virtuales. Comunicar, 42, XXI, 97-105.

García Manzano, A. (2006). Blogs y wikis en tareas educativas. Observatorio Tecnológico del Ministerio de Educación y Ciencia. http://redescolar.ilce.edu.mx/redescolar/lecturas_BB/blog/blogobservatorio.pdf.

González, R. y García, F. (2009). El blog en la docencia universitaria, ¿̇una herramienta útil para la convergencia europea? Relada, 3, 2, 135-144 http: //serviciosgate.upm.es/ojs/index.php/RELADA/article/viewFile/78/78.

González, R.; García, F. y Gonzalo, N. (2011) La creación de una comunidad virtual de aprendizaje a través de los edublog. Relada, 1-13. www.uem.es/myfiles/pageposts/jiu/jiu2010/pdf/9c.pdf

Gros, B. (2007). El diseño de entornos de aprendizaje colaborativo en la enseñanza universitaria. En R. Cabello y D. Levis (Eds.). Medios informáticos en la educación a principios del siglo XXI (pp. 197-215). Buenos Aires: Prometeo. 
Gros, B. (2008). Aprendizajes, conexiones y artefactos. La producción colaborativa del conocimiento. Barcelona: Gedisa.

Hernández, N.; González, M. y Muñoz, P. (2014). La planificación del aprendizaje colaborativo en entornos virtuales. Comunicar, 42, XXI, 25-33.

Huffaker, D. (2005). The educated blogger: using weblogs to promote literacy in the classroom. AACE Journal, 13, 2, 91-98.

Karpova, E.; Correia, A. P. y Baran, E. (2009). Learn to use and use to learn: Technology in virtual collaboration experience. Internet and Higher Education, 12, 45-62. http://dx.doi.org/10.1016/j.iheduc.2008.10.006.

Lara, T. (2005). Blogs para educar. Usos de los blogs en una pedagogía constructivista. Revista Telos. Cuadernos de Comunicación, Tecnología y Sociedad, 65.

http://sociedadinformacion.fundacion.telefonica.com/telos/articulocuaderno.asp@ idarticulo $=2 \& \mathrm{rev}=65 . \mathrm{htm}$.

Levis, D. (2011). Redes educativas 2.1. Medios sociales, entornos colaborativos y procesos de enseñanza y aprendizaje. RUSC, $8,1$. http:/Www.redalyc.org/pdf/780/78017126002.pdf.

López, E. (2009). Innovar con blog en las aulas universitarias. Revista DIM: Didáctica, Innovación y Multimedia, 14.

Marquès, P. (2007). La web 2.0. y sus aplicaciones didácticas. http: //www.peremarques.net/web20.htm.

Merino, J.; López, E. y Ballesteros, C. (2008). El profesor universitario en la sociedad de la información y la comunicación. Revista Espacio y Tiempo. Revista de Ciencias Humanas, 22, 213-231.

Murga, M. A.; Novo, M.; Melendro, M. y Bautista-Cerro, M. J. (2008). Educación ambiental mediante grupos de aprendizaje colaborativo en red: una experiencia piloto para la construcción del EEES. Revista Electrónica Teoría de la Educación. Educación y Cultura en la Sociedad de la Información, 9, 1, 65-77.

Prensky, M. (2001a). Digital Natives, Digital Inmigrants. On the Horizon, NCB University Press, 9, 5.

Prensky, M. (2001b). Digital Natives, Digital Inmigrants, Part II: Do they really think differently? On the Horizon NCB University Press, 9, 6.

Ray, J. (2006). Welcome to the Blogosphere: The educational use of blogs. Kappa Delta Pi Recor, 42, 4, 175-177. http: //dx.doi.org/10.1080/00228958.2006.10518024.

Rebollo, M. ․ A.; García, R.; Buzón, O. y Barragán, R. (2012). Las comunidades virtuales como potencial pedagógico para el aprendizaje colaborativo a través de las Tic. Enseñanza E Teaching, 30, 2, 105-126.

Ruiz, F. (2009). Web 2.0. Un nuevo entorno de aprendizaje en la red. Revista Didáctica, Innovación y Multimedia (DIM), 5, 13. http: //www.pangea.org/dim/revistaDIM13/Articulos/pacoruiz.pdt

Ruiz, M. y Abella, V. (2011). Creación de un blog educativo como herramienta Tic e instrumento TAC en el ámbito universitario. TESI, 12, 4, 53-70.

Salinas, M. I. y Viticcioli, S. (2008). Innovar con blogs en la Enseñanza Universitaria presencial. EDUTEC, Revista Electrónica de Tecnología Educativa, 27.

Sánchez Ambriz, M. (2011). El uso del blog para fomentar el aprendizaje colaborativo en alumnos de maestría. Revista Didáctica, Innovación y Multimedia, 21. http: //www.pangea.org/dim/revista21. 
Scagnoli, N. I. (2005). Estrategias para motivar el aprendizaje colaborativo en cursos a distancia.

http:U/www.ideals.illinois.edu/bitstream/handle/2142/10681/aprendizaje-colaborativoscagnoli.pdf?sequence $=2$

Volman, M. (2005). A variety of roles for a new type of teacher educational technology and the teaching profession. Teaching and Teacher Education, 21, 1, 15-31. http://dx.doi.org/10.1016/j.tate.2004.11.003.

Williams, J. B. y Jacobs, J. (2004). Exploring the use of blogs as learning spaces in the higher education sector. Australasian Journal of Educational Technology, 20, 2, 232-247. 